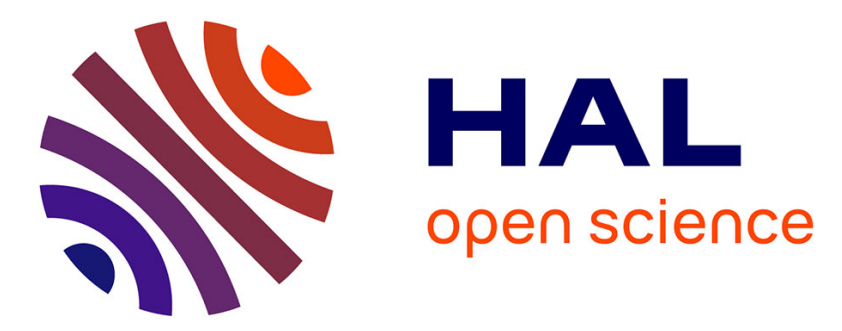

\title{
Le jeu du don et de l'échange. Économie et narcissisme dans La Double Inconstance de Marivaux
}

Christophe Martin

\section{To cite this version:}

Christophe Martin. Le jeu du don et de l'échange. Économie et narcissisme dans La Double Inconstance de Marivaux. Littératures, 1996, 35 (1), pp.87-99. 10.3406/litts.1996.1723 . hal-01759850

\section{HAL Id: hal-01759850 \\ https://hal.science/hal-01759850}

Submitted on 5 Apr 2018

HAL is a multi-disciplinary open access archive for the deposit and dissemination of scientific research documents, whether they are published or not. The documents may come from teaching and research institutions in France or abroad, or from public or private research centers.
L'archive ouverte pluridisciplinaire HAL, est destinée au dépôt et à la diffusion de documents scientifiques de niveau recherche, publiés ou non, émanant des établissements d'enseignement et de recherche français ou étrangers, des laboratoires publics ou privés. 
Article paru dans Littératures, n 35, automne 1996, p. 87-99.

\section{Le jeu du don et de l'échange. Économie et narcissisme dans La Double Inconstance de Marivaux.}

«... vous nous honorez de vos bienfaits, et voilà qui est fini.» La Double Inconstance (acte I, scène 6).

S'agissant de Marivaux, l'on ne parle le plus souvent d'économie que pour signaler l'un des rares éléments biographiques dont on dispose : sa ruine partielle, en 1720, après la banqueroute de Law. Rappelons que cette expérience nouvelle, dont les conséquences furent assurément très profondes sur les esprits, consistait à faire circuler une monnaie de papier gagée par une compagnie de commerce. Par delà les circonstances de son échec, Michel Foucault a montré comment cette expérience ne constituait qu'une étape, fûtelle la plus spectaculaire, dans le mouvement de transition qui fait passer le monde occidental d'une économie où les objets et les êtres échangés possèdent une valeur intrinsèque à celle (baptisée par les historiens «mercantilisme») où le prix des hommes et des choses obéit aux lois d'un marché :

Alors que la Renaissance fondait les deux fonctions du métal monnayé (mesure et substitut) sur le redoublement de son caractère intrinsèque (le fait qu'il était précieux), le XVIIe siècle fait basculer l'analyse; c'est la fonction d'échange qui sert de fondement aux deux autres caractères (l'aptitude à mesurer et la capacité de recevoir un prix apparaissant alors comme des qualités dérivant de cette fonction). ${ }^{1}$

L'hypothèse ici examinée est que l'objet même de La Double inconstance pourrait n'être pas sans rapport avec ce bouleversement qui affecte en profondeur le

\footnotetext{
${ }^{1}$ Michel Foucault, Les Mots et les choses, Paris, Gallimard, 1966, p. 186. Sur l'expérience de Law, voir en particulier les p. 194-196 du chapitre «échanger».
} 
système d'évaluation et le régime des échanges. Le titre de la pièce en constituerait d'ailleurs peut-être un indice : s'il y a double inconstance, ce n'est sans doute pas seulement pour répondre aux exigences d'un code générique (l'un des amants se retrouverait seul, ce qui donnerait au dénouement une amertume difficilement conciliable avec les impératifs de la comédie), mais parce qu'en son principe même, cette comédie s'élabore en fonction du modèle économique de l'échange, qui implique la symétrie d'une double cession et d'une double acquisition.

Car on calcule beaucoup dans La Double Inconstance et, à vrai dire, les personnages ne cessent guère, de manière quasi obsessionnelle tout au long de la pièce, d'estimer la valeur d'échange des êtres et des choses. Une telle perspective économique a d'ailleurs récemment été suggérée par H. Coulet et M. Gilot :

... ce qui explique toutes les réactions [de Silvia et Arlequin] et fonde leur vision du monde, c'est le sentiment et la volonté de connaître le vrai prix des choses, de ne se fier qu'à des valeurs concrètes [...]. Ils sont les champions de l'être contre l'avoir, et des valeurs d'usage contre les valeurs d'échange. ${ }^{2}$

De fait, par la médiation du regard étranger que ses deux héros rustiques portent sur la société de cour, Marivaux offre en spectacle l'étrange «trafic» ${ }^{3}$ dont cette cour est le lieu, et confronte la fragilité, voire la vanité des valeurs qui la fondent aux modestes mais solides bénéfices de l'économie rurale :

LE SEIGNEUR : ... un homme comme moi ne peut demeurer qu'à la Cour, il n'est en considération, il n'est en état de pouvoir se venger de ses envieux qu'autant qu'il se rend agréable au Prince, et qu'il cultive l'amitié de ceux qui gouvernent les affaires.

ARLEQUIN : J'aimerais mieux cultiver un bon champ, cela rapporte toujours peu ou prou, ${ }^{*}$ et je me doute que l'amitié de ces gens-là n'est pas aisée à avoir ni à garder. (II, 5).

Le jeu sur les mots, par le biais de l'antanaclase, n'a en l'occurence rien de gratuit et acquiert même une fonction essentiellement démystificatrice. Car les

\footnotetext{
${ }^{2}$ Notice pour La Double Inconstance in Marivaux, Théâtre complet, éd. établie par Henri Coulet et Michel Gilot, Paris, Gallimard (bibliothèque de la Pléiade), 1993. t. I, p. 853. Toutes les citations de la pièce se réfèrent à cette édition, où la ponctuation et le découpage scénique sont légèrement différents de ceux adoptés par Frédéric Deloffre et Françoise Rubellin pour leur édition du Théâtre complet de Marivaux (Paris, Bordas, 1989).

* Nous soulignons.

${ }^{3}$ Le mot est d'Arlequin, acte II, scène 5.
} 
mots eux-mêmes ont, dans le langage de cour, perdu leur valeur propre, et la pratique du marivaudage 4 vaut dès lors comme dévoilement d'un fauxmonnayage :

LE SEIGNEUR. [...] L'ambition, c'est un noble orgueil de s'élever.

ARLEQUIN. Un orgueil qui est noble! donnez-vous comme cela de jolis noms à toutes les sottises, vous autres? (III, 4).

De même, lorsque le seigneur offre, de la part du Prince, des lettres de noblesse à Arlequin, la réponse de celui-ci prend ironiquement la forme d'un authentique marchandage, seul langage adéquat en ce qu'il manifeste la dégradation des valeurs fondatrices de l'aristocratie, désormais réductibles à des denrées monnayables :

ARLEQUIN : Tenez, accommodons-nous plutôt, ${ }^{*}$ quand on me dira une grosse injure, j'en répondrai une autre, si je suis le plus fort. Voulez-vous me laisser votre marchandise à ce prix-là *? dites-moi votre dernier mot. (III, 4).

La perversion des valeurs est si complète que, conformément à un très ancien topos satirique, la cour devient dans le discours d'Arlequin ce monde à l'envers où «il y a plus de gain à être puni que récompensé» (II, 5).

Il n'est pas certain, pour autant, que les valeurs auxquelles se fient Arlequin et Silvia soient plus «concrètes» que celles définies par le régime des échanges dans la société de cour : bien au contraire, c'est à l'aune d'une valeur on ne peut plus abstraite, à savoir le sentiment amoureux qui les unit, que Silvia et Arlequin estiment les objets matériels (parures, richesses, festins...) et immatériels (prestige, honneurs...) qui leur sont proposés dans les premières scènes de la pièce. D'où l'incompréhension de Trivelin symétriquement exprimée à Silvia :

Qu'est-ce qu'Arlequin au prix* d'un Prince plein d'égards, qui ne veut pas même se montrer qu'on ne vous ait disposée à le voir. (I, 1).

et à Arlequin :

Vous ignorez le prix* de ce que vous refusez. (I, 4).

\footnotetext{
${ }^{4}$ Rappelons que ce terme n'apparait que vers les années 1760. Voir F. Deloffre, Une préciosité nouvelle : Marivaux et le marivaudage, Paris, Belles Lettres, 1955.
} 
C'est bien faute de comprendre le régime de valeurs auquel appartiennent Silvia et Arlequin que Trivelin finit par croire en un «prodige», seule possibilité pour lui de rendre compte de leur obstination à refuser un marché qui, de son point de vue, est évidemment tout à leur avantage. C'est, dira-t-on, que le domestique est tellement corrompu par le système des valeurs de la cour qu'il ne saurait même soupçonner la possibilité de comportements désintéressés. En fait, et là est bien la profondeur de la pièce de Marivaux, La Double inconstance ne confronte nullement des conduites désintéressées à des calculs strictement mercantilistes. Car si Arlequin «s' ennuie de tous les comptes ${ }^{5} »$ de Trivelin, il ne faudrait pourtant pas imaginer que le langage du commerce est le domaine réservé des domestiques du Prince, auquel les deux amants rustiques opposeraient le pur discours du sentiment amoureux. Silvia et Arlequin ne refusent pas les termes du marché, et leur langage en est au contraire profondément imprégné :

LE SEIGNEUR : Je ne m'aperçus donc pas de votre honnêteté ?

ARLEQUIN : Oh que si, mais vous n'aviez point de grâce à me demander, voilà pourquoi je perdis mon étalage*. (II, 5).

SILVIA :Après tout, quel mal y a-t-il qu'il me trouve à son gré ? Prix pour prix $^{*}$, les gens qui nous aiment sont de meilleure compagnie que ceux qui ne se soucient pas de nous, n'est-il pas vrai ? (II, 6).

Mais, au moins dans un premier temps, ils ne peuvent voir en ce marché qui leur est proposé qu'une aberration ${ }^{6}$, puisqu'ils font partie d'un système économique où, contrairement à celui qui règle les échanges à la cour, il existe des valeurs intrinsèques dont certaines sont absolues (en l'occurrence, la fidélité). C'est ce qui se manifeste immédiatement dans la réaction d'Arlequin lorsque Trivelin lui annonce qu'il sait où se trouve Silvia : «Que je suis fâché de n'être pas riche, je vous donnerais tous mes biens pour gages» (I, 4). On pourrait, au premier abord, se demander si cette exclamation est la marque d'un profond désintérêt, ou s'il ne s'agit que de payer Trivelin de mots. L'essentiel est, en fait, qu'Arlequin affirme d'emblée sa croyance en une valeur pour laquelle il est prêt à payer n'importe quel prix. Car, comme telles, les valeurs absolues ne sauraient entrer en comparaison avec les valeurs relatives

\footnotetext{
${ }^{5}$ Acte I, scène 4 : «je commence à m'ennuyer de tous vos comptes». La plupart des éditions modernes corrige le texte et donne «vos contes». On sait qu'étymologiquement, il s'agit du même verbe (computare) mais que les deux mots se sont spécialisés dans des sens différents au XVIe siècle. Or l'ambiguïté est ici précieuse puisqu'avec Trivelin, il n'a effectivement été question que des calculs de l'intérêt.

${ }^{6}$ Silvia : «... Vous a-t-on payés pour [...] me tenir des discours qui n'ont pas le sens commun ?» (acte I, scène 1).
} 
(soumises à de perpétuelles dévaluations et réévaluations) que sont les richesses ou les honneurs :

ARLEQUIN : Vous êtes un grand nigaud, mon ami, de faire entrer Silvia en comparaison avec des meubles, un carrosse et des chevaux qui le traînent... (I, 4).

Contrairement à ce que pourrait laisser penser la réplique d'Arlequin, le marché n'est pas refusé en ce qu'il mettrait en balance des êtres et des choses, mais parce qu'aux yeux des deux amants, il ne peut être considéré que comme un marché de dupes :

TRIVELIN : ... nous avons ici d'aimables filles, épousez-en une, vous y trouverez votre avantage.

ARLEQUIN : Oui-da, que je me marie à une autre, afin de mettre Silvia en colère et qu'elle porte son amitié ailleurs. Oh oh, mon mignon, combien vous a-t-on donné pour m'attraper? Allez, mon fils, vous n'êtes qu'un butor, gardez vos filles, nous ne nous accommoderons pas, vous êtes trop cher. $(\mathrm{I}, 4)$.

SILVIA : On m'ôte mon amant et on me rend des femmes à la place; ne voilà-t-il pas un beau dédommagement? (I, 1).

Mais sans doute faut-il aller plus loin. Si Arlequin et Silvia refusent le marché qui leur est proposé, ce n'est pas (contrairement à ce que croit Trivelin) en dépit de la valeur des offres qui leur sont faites, mais en raison même de leur caractère fastueux :

SILVIA : ... Car c'est un abus que tout ce qu'il fait, tous ces concerts, ces comédies, ces grands repas qui ressemblent à des noces, ces bijoux qu'il m'envoie, tout cela lui coûte un argent infini, c'est un abîme, il se ruine ; demandez-moi ce qu'il y gagne? Quand il me donnerait toute la boutique d'un mercier, cela ne me ferait pas tant de plaisir qu'un petit peloton qu'Arlequin m'a donné. (II, 1).

Il ne s'agit pas seulement ici d'un étonnement critique devant le système des dépenses somptuaires qui est l'une des caractéristiques de l'économie de cour?7. Au premier abord, le discours de Silvia pourrait faire songer à ce que Marcel Mauss désigne sous le nom de «prestation totale» 8 , puisque les dons du Prince

\footnotetext{
7 Voir à ce propos les analyses de Norbert Elias dans La société de cour, Paris, Flammarion, 1985, chapitre II («Le sytème des dépenses»).

8 Voir Marcel Mauss, Essai sur le don. Forme et raison de l'échange dans les sociétés archaïques, in Sociologie et anthropologie, Paris, P. U. F., 1950, p. 143-279.
} 
semblent être essentiellement dispendieux et finalement destructeurs. Mais en réalité, c'est Silvia qui déclare cette dépense improductive et par là-même, la rend telle, puisque l'amour du Prince donne à ses paroles une force quasi performative. Car ces «dons» ne sont en réalité nullement agonistiques : à ce moment de la pièce, ils ne prennent encore sens et valeur que dans l'économie rationalisée d'un marché. C'est dire en fait que ces «dons» n'en sont pas vraiment, dans la mesure où ils sont effectués en vue d'un gain immédiat et d'une fin bien précise : obtenir de Silvia un don de soi au Prince, et d'Arlequin le renoncement à ce bien qu'est Silvia. Celle-ci ne se prive pas d'accuser la logique mercantiliste de cette «générosité»: «le Prince me veut en troc, et jamais nous ne finirons ce marché-là.» (II, 3). Ce qui rend cette transaction impossible, ce n'est pas seulement l'amour de Silvia et d'Arlequin mais les termes mêmes dans lesquels ce marché leur est proposé : la seule chose que les deux amants peuvent céder, ce n'est pas Silvia elle-même (valeur absolue, donc incessible et inéchangeable) mais seulement son corps. L'hypothèse est d'ailleurs envisagée par Arlequin mais il montre fort bien qu'elle ne serait à nouveau qu'un marché de dupes où, cette fois, personne ne trouverait son compte : «il n'aurait que la femme, moi j'aurais le cœur, il nous manquerait quelque chose à l'un et à l'autre, et nous serions tous trois mal à notre aise» $(\mathrm{I}, 4)$.

Pour les deux jeunes gens, le refus est donc d'autant plus impérieux qu'il est la seule possibilité de n'être pas traités à quelque degré comme des choses, réduits à l'état de marchandises que l'on achète, ce qui entrainerait la perte de toute valeur à leurs propres yeux. Le faste du Prince exerce donc une contrainte qui menace non seulement le lien qui les unit mais la qualité intrinsèque de leur être : d'une part, ses dons exigent en réalité une contrepartie ; d'autre part, et de manière absolument indissociable, ils manifestent l'écrasante supériorité du Prince, conformément à un principe dégagé par M. Mauss ${ }^{9}$. Notons à ce propos que le choix par le Prince d'une simple bergère pourrait n'être pas seulement lié à une nostalgie aristocratique pour des formes idéalisées de la vie pastorale, mais aussi, comme l'a souligné R. Démoris, au fait que «le choix paradoxal comporte [...] une appréciable gratification narcissique, puisqu'il renvoie au Prince une image de sa propre supériorité... » ${ }^{10}$. En exhibant cette supériorité, les dons du Prince ne peuvent, inversement, que renvoyer Silvia à sa propre infériorité, selon un mécanisme que Marivaux a analysé quelques années plus tard dans le premier livre de La Vie de Marianne, à propos des équivoques de la charité :

\footnotetext{
9 «Donner, c'est manifester sa supériorité», M. Mauss, op. cit., p. 269.

10 René Démoris, Lecture de Les Fausses Confidences de Marivaux. L'être et le paraître, Paris, Belin, 1987, p. 27.
} 
Les bienfaits des hommes sont accompagnés d'une maladresse si humiliante pour les personnes qui les reçoivent ! [...] qu'est-ce qu'une charité qui n'a point de pudeur avec le misérable, et qui, avant que de le soulager, commence par écraser son amour-propre. ${ }^{11}$

Cette menace que les dépenses princières font peser sur le sentiment de leur propre valeur est parfaitement perçue par Silvia et Arlequin, ainsi qu'en témoigne, une nouvelle fois, la symétrie de leurs répliques :

SILVIA : Force-t-on les gens à recevoir des présents malgré eux ? (I, 1).

ARLEQUIN : Qui est donc cet original-là, qui me donne des valets malgré moi? (I, 4).

SILVIA : ... pourvu que [le Prince] ne me dise pas après : Pourquoi as-tu pris mes présents? (II,3).

ARLEQUIN : J'ai peur d'être en pension sans le savoir. (III, 2).

Reste à expliquer comment Flaminia parvient à leur faire réévaluer non pas vraiment les mêmes objets que ceux qu'ils ont rejetés avec mépris (c'est-àdire en refusant de leur accorder le moindre prix) dans les premières scènes, mais du moins les biens dont ils considéraient alors qu'ils avaient une valeur absolue. Pour faire basculer Silvia et Arlequin dans le régime de l'échange et dans la circulation des désirs et des richesses, il faut d'abord que Flaminia atteigne le fondement même de toute Valeur. Fondement qu'en bonne courtisane, elle ne peut ignorer puisque tous les moralistes classiques l'ont désigné avant elle, sous le nom d'amour-propre, de vanité, ou de concupiscence. Que l'on se rappelle, par exemple, cette maxime de La Rochefoucauld : «La flatterie est une fausse monnaie qui n'a cours que par notre vanité»; ou ce fragment dans lequel Pascal affirme que la concupiscence fait du plaisir «la monnaie pour laquelle nous donnons tout ce qu'on veut». ${ }^{12}$ L'originalité de Marivaux est d'avoir, très tôt, tenté d'appliquer cette analyse (d'origine sans doute augustinienne) aux différentes techniques du commerce dans leurs aspects les plus quotidiens, ainsi qu'en témoigne un passage (paru pour la première fois dans le Mercure en 1717) des Lettres sur les habitants de

11 Marivaux, La Vie de Marianne, éd. Frédéric Deloffre, Paris, Garnier, 1963, p. 29.

12 La Rochefoucauld, Réflexions ou sentences et maximes morales, Paris, 1665, maxime $\mathrm{n}^{\circ} 158$. Pascal, Pensées, éd. Brunschvicg, fr. 24. Voir sur ce point les analyses de Pierre Force dans Molière ou le prix des choses, Morale, économie et comédie, Paris, Nathan, 1994. 
Paris décrivant «l'art de captiver la bienveillance» chez les marchandes de mode :

Êtes-vous belle et jeune ? [Ces marchandes] vous cajolent sur vos appas en déployant leurs marchandises : ces compliments ne sont pas étrangers à la vente; on dirait qu'ils font partie de la marchandise même.* [...] Êtes-vous jeune homme? Elles font en sorte qu'un peu de galanterie vous amuse. ${ }^{13}$

Même si elle est ici infiniment moins subtile, cette captatio benevolentiæ n'est pas d'une nature différente de celle qui fonde la stratégie de Flaminia. Car, en flattant l'orgueil et la vanité, il s'agit dans les deux cas de captiver le désir de l'autre, c'est-à-dire de rendre celui-ci captif de l'objet même de la transaction. Et c'est bien l'ignorance de ce principe qui rend les offres de Trivelin au début de la pièce non seulement inefficaces mais profondément anti-économiques puisqu'en blessant l'amour-propre de Silvia et d'Arlequin, il ne fait que renforcer le lien qui les unit et garantit à leurs yeux leur propre valeur.

C'est bien pourquoi aussi les flatteries de Flaminia ne visent que secondairement à s'attirer la bienveillance des deux amants. Les éloges dont elle les gratifie ont en réalité de tout autres enjeux :

FLAMINIA : Eh ma chère enfant, avons-nous rien ici qui vous vaille*, rien qui approche de vous? (II, 1).

FLAMINIA : Pardi, qui est-ce qui ne vous plaindrait pas ? qui est-ce qui ne s'intéresserait pas à vous? vous ne connaissez pas ce que vous valez*, Arlequin.

ARLEQUIN : Cela se peut bien, je n'y ai jamais regardé de si près. (II, 4).

Ce langage a pour fonction essentielle d'intégrer les deux héros de la campagne, et presque à leur corps défendant, au système des valeurs de la cour, système économique où les objets et les personnes n'ont pas de valeur en soi, mais où c'est en fait le Prince qui détermine le «prix» des êtres et des choses, comme le chevalier l'apprend à Arlequin dans l'acte II, et ainsi que l'a montré Norbert Elias :

La disgrâce royale [...] pouvai[t] menacer à tout moment les sources de revenus d'un courtisan, le niveau de vie de sa famille, son prestige, sa «valeur marchande» dans la société de cour. ${ }^{14}$

13 Lettres sur les habitants de Paris, in Journaux et CEuvres diverses, éd. F. Deloffre et M. Gilot, Paris, Garnier, 1988, p. 17.

14 N. Elias, op. cit., p. 269. 
Cette loi économique est, plus particulièrement, le fondement même du système de Law, tel que Turgot en a fait la critique : «la monnaie n'est qu'une richesse de signe dont le crédit est fondé sur la marque du Prince». ${ }^{15}$ En feignant d'entrer dans leurs intérêts, il s'agit donc moins pour Flaminia de se faire valoir auprès de Silvia et d'Arlequin, que de faire valoir ces derniers auprès de ceux et celles qui composent la cour. Ainsi, après l'échec de sa tentative de séduction sur Arlequin, Lisette n'est plus que l'instrument d'une valorisation de Silvia, qui permet l'adhésion de celle-ci au régime des valeurs relatives :

SILVIA : ... chacun a son prix, ne semble-t-il pas que je ne vaille pas bien ces femmes-là ? je ne voudrais pas être changée contre elles. (II, 3).

Quant au seigneur qui vient voir Arlequin pour lui demander d'intercéder en sa faveur auprès du Prince, il est le moyen que Flaminia se donne pour inciter le jeune villageois à se persuader de sa valeur et de son «crédit» :

LE SEIGNEUR : Je vous ai beaucoup d'obligation, j'attends l'effet de vos promesses : adieu, monsieur Arlequin.

ARLEQUIN : Je suis votre serviteur; diantre, je suis en crédit car on fait ce que je veux. (II, 5).

Dès lors, la possibilité de l'échange devient pensable pour les deux âmes rustiques, fût-ce sur le mode de l'irréel du passé :

SILVIA : Tenez, si j'avais eu à changer Arlequin contre un autre, ç'aurait été contre un officier du palais, qui m'a vue cinq ou six fois, et qui est d'aussi bonne façon qu'on puisse être : il y a bien à tirer si le Prince le vaut; c'est dommage que je n'aie pu l'aimer dans le fond, et je le plains plus que le Prince. (II, 1)

ou du conditionnel :

ARLEQUIN : ... il est fâcheux que j'aime Silvia, sans cela je vous donnerais de bon cœur la ressemblance de votre amant. (II, 5).

Mais pour que Silvia et Arlequin acceptent d'entrer pleinement dans le règne des valeurs relatives, il faut aussi qu'ils soupçonnent (sans forcément se l'avouer) que ce qu'ils prenaient pour une valeur absolue (et qu'ils incarnaient

15 Tugot, Seconde lettre à l'abbé de Cice, 1749. C'était déjà l'analyse de Scipion de Grammont : «La monnaie n'emprunte point sa valeur de la matière dont elle est composée, mais bien de la forme qui est l'image ou la marque du Prince.» (Le Denier royal, traité curieux de l'or et de l'argent, Paris, 1620, p. 13-14). Ces deux documents sont cités par M. Foucault, op. cit., p. 187 et p. 195. 
chacun à l'égard de l'autre) n'étaient pas à proprement parler une valeur, mais seulement un bien, c'est-à-dire un présent de la nature :

SILVIA : Mettez-vous à ma place : c'était le garçon le plus passable de nos cantons, il demeurait dans mon village, il était mon voisin... (II, 7).

Car «avant l'échange, il n'y a que cette réalité, rare ou abondante, que fournit la nature. Seule la demande de l'un, et la renonciation de l'autre sont capables de faire apparaître des valeurs» ${ }^{16}$. D'où pour Silvia les plaisantes contradictions de la scène $7 \mathrm{du}$ dernier acte :

SILVIA : [...] j'ai peur qu'Arlequin ne s'afflige trop, qu'en dites-vous ? mais ne me rendez pas scrupuleuse.

FLAMINIA: Ne vous inquiétez pas, on trouvera aisément moyen de l'apaiser.

SILVIA avec un petit air d'inquiétude: De l'apaiser ! diantre il est donc bien facile de m'oublier à ce compte ? est-ce qu'il a fait quelque maîtresse ici ?

FLAMINIA : Lui vous oublier! j'aurais donc perdu l'esprit si je vous le disais ; vous serez trop heureuse s'il ne se désespère pas.

SILVIA : Vous avez bien affaire de me dire cela; vous êtes cause que je redeviens incertaine, avec votre désespoir.

Où l'on voit que le désir du «chevalier» ne constitue pas pour Silvia un gage suffisant de sa propre valeur : encore faut-il qu'il en coûte à Arlequin de renoncer à elle. Mais pour conserver le sentiment de cette même valeur, il faut aussi que cette renonciation soit sans désespoir sous peine de la dévaluer à ses propres yeux comme ingrate et cruelle. Car le fondement de toute valeur est la gratification narcissique que le sujet retire non seulement de l'acquisition de l'objet, mais aussi de sa cession.

Que l'amour-propre soit l'unique origine du prix accordé aux êtres et aux choses, c'était déjà, on l'a dit, la leçon de La Rochefoucauld et de Pascal. Mais la nouveauté que La Double inconstance introduit, selon nous, dans cette analyse de la constitution de la valeur, c'est l'idée d'un bénéfice narcissique qui, paradoxalement, n'est jamais aussi gratifiant que lorsque l'échange prend la forme, sublime, de la renonciation ou celle, aristocratique, du don généreux. À cet égard, on peut rappeler l'anecdote rapportée à la fin de la vingt-troisième feuille du Spectateur français : chez un marchand d'habits réputé âpre au gain, le rédacteur se comporte d'une manière propre à stupéfier l'ami qui l'accompagne en laissant le marchand se servir dans sa bourse sans même lui demander le

${ }^{16}$ M. Foucault, op. cit., p. 205. 
prix de l'étoffe. Le marchand ne se montre nullement «avare» et voici l'explication que le rédacteur donne de ce paradoxe :

... je l'ai d'un coup tellement pénétré des honneurs que lui prodiguaient ma confiance, je l'ai rendu si vain du portrait flatteur qu'elle lui faisait de lui-même, que la tête lui en a tourné d'orgueil et de reconnaissance, et dans la chaleur de ces mouvements-là, passionné comme il était du plaisir d'être pris pour un si galant homme, hélas, il s'est laissé mené comme j'ai voulu, voilà tout ce que c'est. ${ }^{17}$

Ce «coup de baguette», ainsi que l'appelle le rédacteur, n'est au fond que le retournement sur le marchand de la captation d'amour-propre décrite par la Lettre sur les habitants de Paris citée précédemment. Et la profondeur de l'analyse marivaudienne est de détecter ce qui, dans le régime marchand, révèle la prééminence d'investissements affectifs et relève, on le verra, de l'économie archaïque du don.

Ce qui fait l'objet et la valeur de la négociation, c'est la surévaluation narcissique qu'elle permet. Ce qu'on acquiert dans l'échange, c'est une valorisation de soi. Cette loi se vérifie, dans La Double inconstance, dès l'instant où Flaminia incite le Prince à renoncer (au moins en apparence, et c'est bien là toute l'ambiguiité) à la contrepartie implicitement exigée par le faste dont il entoure Silvia et Arlequin. Et c'est bien pourquoi il est si important que le Prince reste incognito le plus longtemps possible. Il s'agit en fait de dissocier théâtralement le donateur et celui qui désire bénéficier du contre-don, sous la forme de l'amour de Silvia. Car, en vertu du principe de la gratification narcissique, le don sans réserve et sans contrepartie est le seul moyen $\mathrm{d}^{\prime}$ «obliger» le donataire, et d'en obtenir un don réciproque. L'économie marivaudienne se fonde ici sur une loi que M. Mauss a analysée comme l'une des plus fondamentales dans l'économie du don, à savoir l'«obligation de rendre le présent reçu» ${ }^{18}$. Encore faut-il s'entendre sur le sens du mot «obligation»: tant que les bienfaits du Prince exigeaient, de manière plus ou moins implicites, un contre-don, ils supposaient la recherche d'un contrat liant les deux parties, et l'on a vu que c'était précisément ce qui les rendaient inopérants. Alors que le don proprement dit se définit au contraire en ce qu'il refuse explicitement l'échange, en ce qu'il nie la réciprocité et s'affirme sans contrepartie. Toutefois, cela ne signifie nullement que cette contrepartie ne se réalise pas, mais bien plutôt que le contre-don ne se réalise qu'en fonction de

\footnotetext{
17 Marivaux, Le Spectateur français, vingt-troisième feuille [8 janvier 1724], in Journaux et OEuvres diverses, op. cit., p. 252.

18 M. Mauss, op. cit., p. 153.
} 
cette négation même. C'est dans la dynamique du don que réside l'obligation ${ }^{19}$ du contre-don et nullement dans un contrat préalable, fût-il tacite. Ainsi, (et sans exclure évidemment les éventuels agréments de sa personne) c'est à proportion même du «désintéressement» $d u$ «chevalier» que Silvia est prête à lui «rendre le change» pour reprendre ses propres termes 20 ; phénomène qu'elle analyse avec un mélange de naïveté profonde et de subtile ironie :

Comment voulez-vous que je vous renvoie. Vous vous tairez, s'il me plaît; vous vous en irez, s'il me plaît; vous n'oserez pas vous plaindre, vous m'obéirez en tout. C'est bien là le moyen de faire que je vous commande quelque chose. (II, 9).

Si bien que dans son esprit, la dissociation avec la figure invisible du Prince n'en est que plus radicale : «... voilà le Prince qui demande mon cœur, voilà vous qui méritez de l'avoir».

Mais la scène décisive à cet égard, c'est naturellement la grande confrontation d'Arlequin et du Prince, au milieu du troisième acte : non seulement parce que c'est dans cette longue négociation que s'organise la recomposition des duos et que sont scellés les termes de l'échange, mais parce que c'est ici que se vérifie pleinement la logique paradoxale de l'économie marivaudienne. Il faut dire qu'à ce moment de la pièce, tout n'est pas encore acquis ; car en dévoilant sa véritable identité à Arlequin, le Prince ne peut manquer d'être à nouveau confronté à l'obstacle de sa supériorité. Autrement dit, en imposant à Arlequin un marché qui ne laisse à ce dernier aucun bénéfice narcissique, il en revient au blocage initial du commerce amoureux, obligeant le jeune villageois à se replier sur une économie autarcique du bien, lors même qu'il est, plus ou moins consciemment, déjà détaché de Silvia :

ARLEQUIN : Que voulez-vous, Monseigneur ? j'ai une fille qui m'aime, vous, vous en avez plein votre maison, et nonobstant vous m'ôtez la mienne ; prenez que je suis pauvre, et que tout mon bien est un liard, vous qui êtes riche de plus de mille écus, vous vous jetez sur ma pauvreté et vous m'arrachez mon liard, cela n'est-il pas bien triste?

$[\ldots]$

LE PRINCE : Je te prive de Silvia, il est vrai : mais demande-moi ce que tu voudras, je t'offre tous les biens que tu pourras souhaiter, et laisse-moi cette seule personne que j'aime.

19 La langue classique connait d'ailleurs parfaitement cette acception («obliger quelqu'un», «être obligeant»...) et l'on en trouve plusieurs occurrences dans La Double Inconstance.

20 Acte II, scène 3. Notons toutefois que le «chevalier» trahit in extremis le souci qu'il a de son propre intérêt : «SILVIA : ... je vous aimerai s'il me plaît, sans qu'il y ait le petit mot à dire. LE PRINCE : Je n'y gagne rien, car il ne vous plaît point.» (III, 8). 
ARLEQUIN : Ne parlons point de ce marché-là, vous gagneriez trop sur moi. [...]. (III, 5).

En revanche, c'est au moment exact où le Prince en vient à renoncer aux termes de ce marché, tout en maintenant l'offre de ses dons (lesquels deviennent désormais sans contrepartie) qu'Arlequin finit, à demi-mot, par lui céder Silvia

LE PRINCE : ... j'espérais quelque chose de ton cœur pour moi, je t'aurais eu plus d'obligation que je n'en aurai jamais à personne : mais tu me fais tout le mal qu'on peut me faire ; va, n'importe, mes bienfaits t'étaient réservés, et ta dureté n'empêchera pas que tu n'en jouisses* [...] sois heureux aux dépens *de tout mon repos.

ARLEQUIN : Vous avez tant de charité pour moi, n'en aurais-je donc pas pour vous?

LE PRINCE triste : Ne t'embarrasse pas de moi.

ARLEQUIN : Que j'ai de souci ! le voilà désolé.

LE PRINCE en caressant Arlequin: Je te sais bon gré de la sensibilité où je te vois : adieu, Arlequin, je t'estime malgré tes refus.

ARLEQUIN laisse faire un ou deux pas au Prince: Monseigneur.

LE PRINCE : Que me veux-tu ? me demandes-tu quelque grâce?

ARLEQUIN : Non, je ne suis qu'en peine de savoir si je vous accorderai celle que vous voulez. (III, 5).

On aurait tort, sans doute, de penser que l'affrontement des volontés contradictoires cède peu à peu la place à un souci d'accommodement où, tour à tour, chacun cèderait sur son désir. Le combat continue, mais il prend désormais une autre forme : les protagonistes font maintenant assaut de générosité, à coup de dons qui en eux-mêmes ne veulent pas être rendus parce qu'ils sont pour chacun le moyen d'être reconnu par l'autre comme une valeur au moins égale, et peut-être supérieure. Combat difficile, parce que les exigences de l'amour-propre sont contradictoires : pour le Prince, céder devant un simple villageois serait presque déroger («... je descends jusqu'à te prier de me céder Silvia de bonne volonté») ; mais ne pas céder reviendrait aussi à se rendre indigne de sa noblesse et de son rang ainsi qu'Arlequin le lui signifie fermement en une stratégie qui rappelle le «coup de baguette» magique transformant l'avide marchand en gentilhomme généreux dans la Lettre citée plus haut: «Je sais que vous êtes un bon prince... ». Pour Arlequin, dira-t-on, le renoncement est d'autant plus facile qu'il est avant tout soucieux de retrouver Flaminia. Mais ce serait oublier que l'infériorité de son rang donne à ce combat un enjeu peut-être plus essentiel encore, dans la mesure où il exaspère le dilemme de l'amour-propre : car céder Silvia peut, dans un premier temps, être perçu comme un signe d'allégeance, et donc un aveu de moindre valeur ; mais, 
plus profondément, c'est aussi, pour ce petit villageois, le seul moyen de se hisser au niveau du Prince et, finalement, de manifester sa supériorité.

Tant que le don se présente sous la forme d'une négociation, l'amour-propre ne permet pas à Arlequin de l'accepter. Mais dès que les dons du Prince ne réclament plus leur contrepartie, c'est ce même amour-propre qui l'«oblige» et, en un sens, lui interdit le refus.

La Double Inconstance nous semble donc re-présenter (à tous les sens du terme) l'intégration progressive des deux jeunes gens de la campagne au règne des valeurs relatives. Mais pour que ce passage soit possible, il a fallu l'avènement, ou plutôt la redécouverte d'une économie plus archaïque, celle du don réciproque, dont la fonction est essentiellement intégratrice : c'est sur le don que se fonde la possibilité de l'échange. Mais l'existence de cette économie au sein du théâtre de Marivaux ne saurait manquer, semble-t-il, de poser à nouveau l'éternelle question du rôle de la sincérité dans son œuvre : s'il suffit de refuser la contrepartie pour l'obtenir, rien, apparemment, ne devrait être plus simple que de feindre ce refus. La réussite de Flaminia ne tient-elle pas à la connaissance de cette loi, et à son utilisation stratégique ? À s'en tenir là, on risquerait pourtant de méconnaître la portée du théâtre marivaudien : si la stratégie de Flaminia est une condition nécessaire au processus de l'échange, on ne saurait, pour autant, affirmer avec certitude qu'elle est suffisante. La Double Inconstance se fonde en fait sur un principe peut-être plus profond et plus obscur, qui n'est sans doute pas sans rapport avec l'apprentissage de la perte : «J'ai peur de vous perdre» disait Silvia à Arlequin au début de la pièce ${ }^{21}$. C'est seulement à surmonter cette peur qu'elle peut accéder au règne des valeurs, où certes, il n'existe pas de juste prix, mais où la possibilité d'être considéré comme précieux est, en droit, infinie. En fait, chacun des protagonistes de la pièce doit, à des degrés divers, en passer par cette expérience pour devenir à la fois sujet et objet de désir. Et à ce jeu du «qui perd gagne», il n'est pas certain que l'on puisse tricher.

${ }^{21}$ Acte I, scène 8. 
